\title{
Comparative Analysis of DWT and DCT Image Compressions Techniques using VHDL
}

\author{
Arvind Kumar \\ Assistant professor \\ Electronics and Communication \\ Engineering \\ I.T. Gopeshwar Chamoli \\ Uttarakhand
}

\author{
Arun Uniyal \\ Assistant professor \\ Electronics and Communication \\ Engineering \\ I.T. Gopeshwar Chamoli \\ Uttarakhand
}

\author{
Pawan Kumar \\ Assistant professor \\ Computer Science and \\ Engineering \\ I.T. Gopeshwar Chamoli \\ Uttarakhand
}

\begin{abstract}
Image compression is very important part of the digital image processing. Image compression is the method of reducing the size of image, which is helpful to increase the storage and transmission performance. In the compression method not only concentrate on reducing the size of image, but also concentrate on the quality and information of the image. Image compressions have more researched area, and many compression standards are place. But still here there is a scope for high compression quality; the JPEG and JPEG2000 standard make use of Discrete cosine transform DCT and Discrete Wavelet transform DWT for compression respectively. Discrete wavelet and Discrete cosine transforms are the common method used in image compression. Wavelet transform are very powerful as compared to Fourier transform, because it have ability to describe any type of signals both in time and frequency domain simultaneously. In this thesis two image compression methods are simulated, the first method is based on Discrete Wavelet transform (DWT), and second method is based on Discrete Cosine transform (DCT). The aim of the thesis as to compress an image using DWT and DCT encoding method, to implement DWT and DCT using VHDL and measure the performance in terms of memory utilization and timing parameters. The first part of the thesis presents an implementation of Discrete Wavelet Transform with HAAR Wavelet and the second part of the thesis presents an implementation of Discrete Cosine Transform. The performance will be measured in term of memory utilization and timing parameters.
\end{abstract}

\section{General Terms}

Discrete Wavelet transform, Discrete Cosine transform, VHDL.

\section{Keywords}

DWT, DCT, VHDL, JPEG2000, etc

\section{INTRODUCTION}

The expansion of transmission technology over the last two decades. The demand for digital information increases dramatically. The advances in technology have created the utilization of digital image prevailing to an outsized extent. Still images are widely used in application like medical and satellite images. Digital image are comprised of an enormous

amount of data. Reduction in the size of image data for both storing and transmission of digital images are becoming increasingly important as they find more application. Image

Compression is a mapping from a higher dimensional space to a lower dimensional space. Image compression play important role in many multimedia application, such as image storage and transmission. The basic goal of image compression is to represent an image with minimum number of bits of an acceptable image quality. All image compression algorithms strive to remove statistical redundancy and exploit perceptual irrelevancy while reducing the amount of data as much as possible. Data compression or image compression is the technique to reduce the redundancies in data representation in order to decrease data storage requirements and hence its coast of communication. Decreasing the storage space requirement is equivalent to increasing the capacity of the storage medium and also increases communication bandwidth. Then the development of efficient compression techniques will continue to be a design challenge for future communication systems and advanced multimedia application.

Image is represented by a combination of pixels and redundancy. A pixel is the portion of image that must be preserved permanently in its original form in order to correctly interpret the meaning or purpose of the image (data). Redundancy bits are the portion of image that can be removed when it is not needed or can be reinserted to interpret the image (data) when needed. Most often, the redundancy is reinserted in order to generate the original data (image) in its original form. The method to reduce the redundancy bits of image (data) is defined as image (data) compression. The redundancy bits in an image (data) representation are reduced such a way that it can be subsequently reinserted to recover the original image, and which is called decompression of the data

\subsection{Need for Image- Compression}

The amount of data transmitted through the Internet doubles every year, and large portion of that data comprises of image reducing the band width need of any given device will result in significant cost reduction and will make the use of the device more affordable. Image compression offers ways to represent an image in a more compact way, so that image can be stored in a compact manner and can be transmitted faster.

\subsection{Performance Parameters}

In this paper to reduce the size of the image and comparative analysis of these two methods which are the best methods according to time analysis and memory analysis.

(1) In DWT image compression, first split the image (because image is a group of pixel) in discrete form and represent it histogram representation. Then used HAAR DWT algorithm and find the simulation results in VHDL Simulator. 
(2) Similarly, In DCT image compression, first split image and represent it histogram according to image pixels in $\mathrm{x}$-axis and $\mathrm{y}$-axis. Then represent it DCT matrix representation according to DCT algorithm, after DCT algorithm find the result of the image compression in VHDL Simulator.

\section{DWT IMAGE CODING}

The Discrete Wavelet Transform (DWT) is based on sub-band coding, it is found to yield a fast computation of Wavelet Transform. Discrete Wavelet Transform is easy to implement and reduces the computation time. In Continues Wavelet Transform, the signals are analyzed using a set of basic functions which relate to each other by simple scaling and

translation or shifting. In the case of DWT, time-scale representation of the digital signal is obtained using digital filtering method. The signal to be analyzed is passed through filters with different cutoff frequencies at different scales.

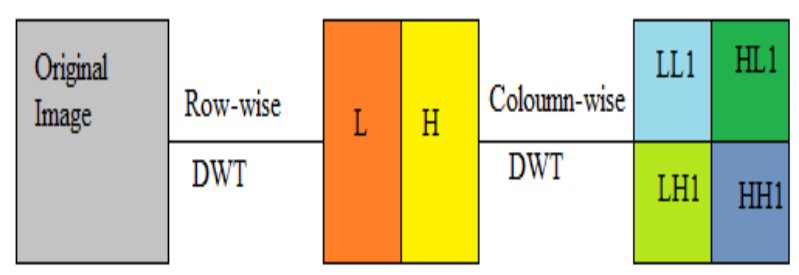

(a) First level Decomposition

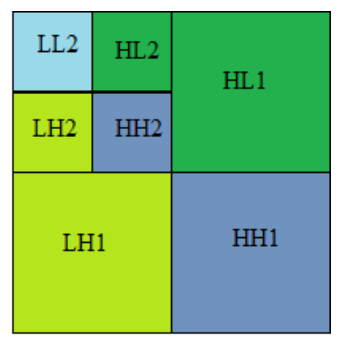

(b) Second level Decomposition

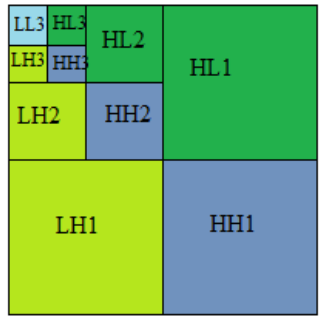

(c) Third level Decomposition

Figure 1.1 Row-column computation of 2-D DWT

Every pixels of the 2D image have own $\mathrm{x}$-axis and $\mathrm{y}$-axis, so we will represent the image pixels in histogram representation. After then the image will be applying to a filter bank, the filter bank will consist of Low-pass and Highpass filters, then the image signal will be separated high band signal and low band signal, according the HAAR DWT algorithm the low band and high band image signal have four possible combination, such as LL,LH,HL,HH. The LL band is more significant band it contains more information of the original image, so it is most important part of the algorithm process. The LL band again sub divided to lower band till to the desired output will not obtained, this process shown below in the figure1.1
In figure 1.1 there are shown a resulted image after applying encoding process. In this figure are four blocks, the first half upper block show the approximation, and second upper half block show the horizontal detail. First lower level block shows vertical detail and second lower level block shows diagonal detail. In this algorithm is shown one level discrete wavelet transform. By applying this process more than one time it can increase the level of DWT. Second and third level DWT gives the better compression ratio of image. But it will come with loss of some information

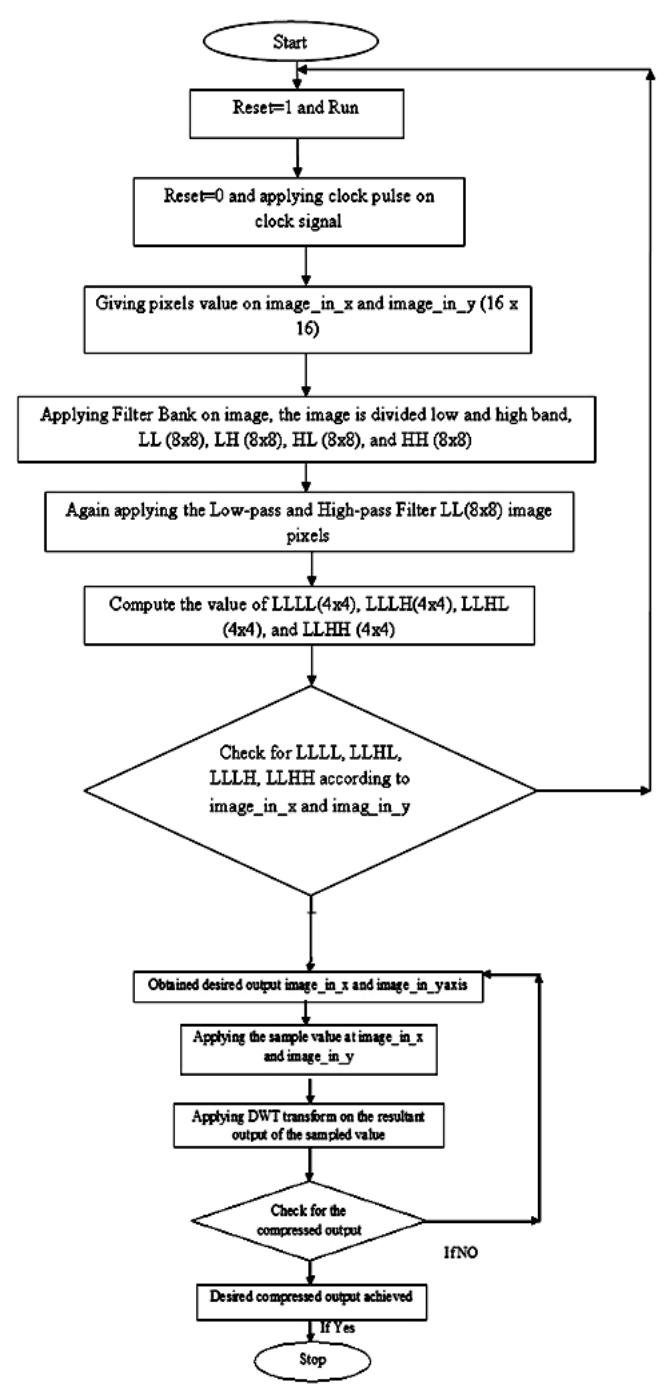

Figure1.2: DWT Algorithm for Image Compression

\section{DCT IMAGE CODING}

A discrete cosine transform consist of a set of basis vector that are sampled cosine function. DCT is a technique for converting a signal into elementary frequency components and it is widely used in image compression.

The general formula of a Discrete Cosine Transforms is given by

Where 
$\mathrm{G}(\mathrm{y})$ is the calculated DCT coefficient

$\mathrm{F}(\mathrm{v})$ is the input data value

Matrix representation of the Forward Discrete Cosine Transform

$$
G(y)=\sum_{v=0}^{7} F(v) \cos \left[\left(\frac{2 y+1}{16}\right) v \pi\right]
$$

Then the matrix representation of Forward Discrete Cosine Transform (FDCT) is given by.

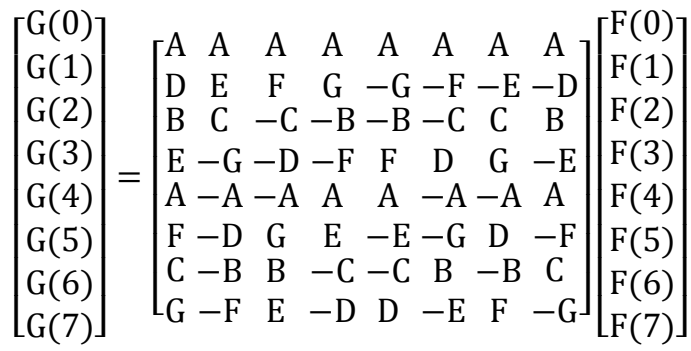

$$
\begin{aligned}
& {[\mathrm{G}]=[\mathrm{M}][\mathrm{F}]}
\end{aligned}
$$

Where

$\mathrm{A}=\operatorname{Cos}($ ? /4) $\mathrm{B}=\operatorname{Cos}(? / 8) \mathrm{C}=\operatorname{Sin}(? / 8) \mathrm{D}=\operatorname{Cos}(? / 16)$

$\mathrm{E}=\operatorname{Cos}(3 ? / 16) \mathrm{F}=\operatorname{Sin}(3 ? / 16) \mathrm{G}=\operatorname{Sin}(? / 16)$

G Forward DCT coefficients matrix

$F$ Input values matrix

The values A, B, C, D, E, F, and G of the matrix M were extracted from the general formula. Matrix of the Inverse Discrete Cosine Transform (IDCT)

$$
\left[\begin{array}{l}
G(0) \\
G(1) \\
G(2) \\
G(3) \\
G(4) \\
G(5) \\
G(6) \\
G(7)
\end{array}\right]=\left[\begin{array}{cccccccc}
A & D & B & E & A & F & C & G \\
A & E & C & -G & -A & -D & -B & -F \\
A & F & -C & -D & -A & G & B & E \\
A & G & -B & -F & A & E & -C & -D \\
A & -G & -B & F & A & -E & -C & +D \\
A & -F & -C & D & -A & -G & B & -E \\
A & -E & C & G & -A & D & -B & F \\
A & -D & B & -E & A & -F & C & -G
\end{array}\right]\left[\begin{array}{l}
F(0) \\
F(1) \\
F(2) \\
F(3) \\
F(4) \\
F(5) \\
F(6) \\
F(7)
\end{array}\right]
$$

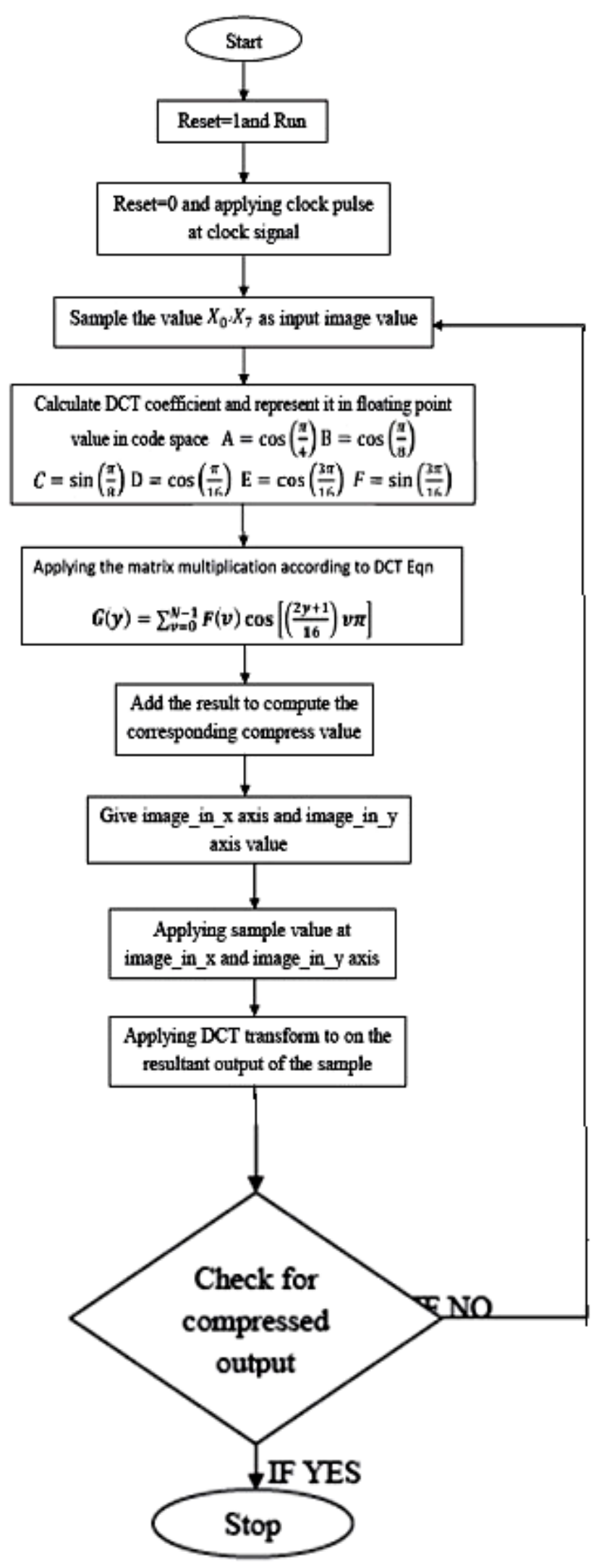

Figure 1.3 Flow Chart of DCT Algorithm for Image Compression using VHDL 


\section{DWT AND DCT OUTPUT OF IMAGE COMPRESSION RESULTS IN VHDL}

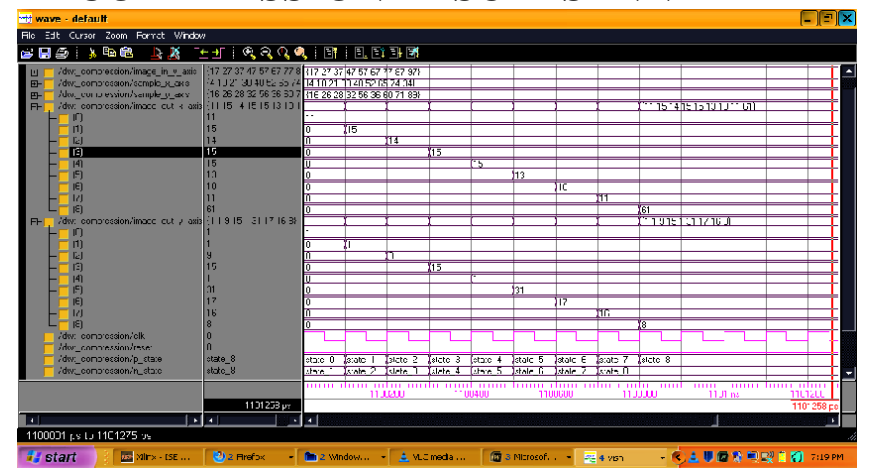

Figure 1.4 Modelsim output of compressed DWT using HAR DWT

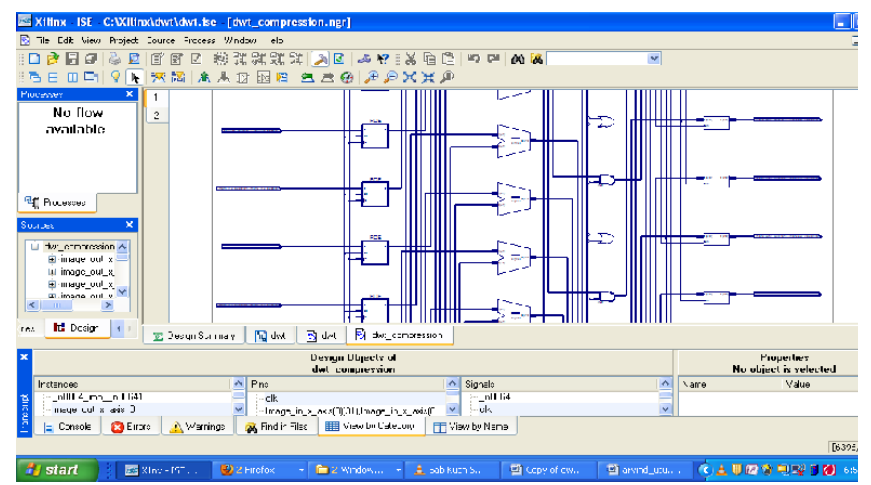

Figure 1.5 Internal schematic of compressed DWT

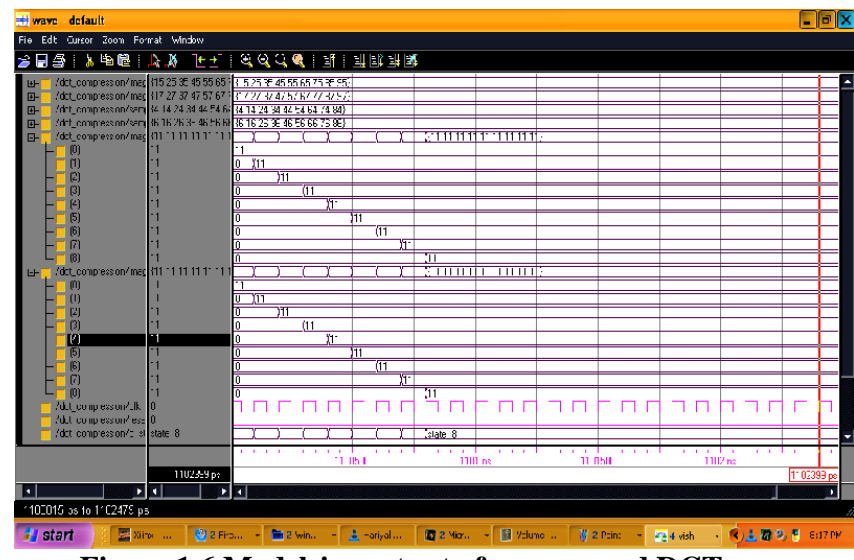

Figure 1.6 Modelsim output of compressed DCT

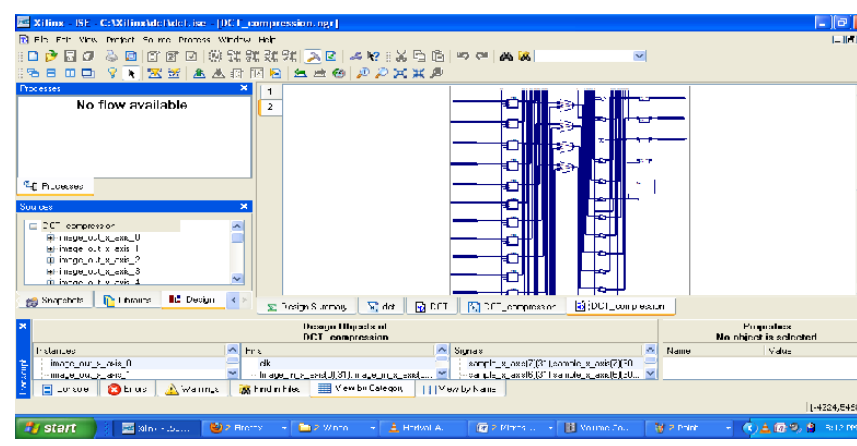

Figure 1.7 Internal schematic of compressed DCT
5. COMPARATIVE ANALYSIS OF DWT AND DCT IMAGE COMPRESSION

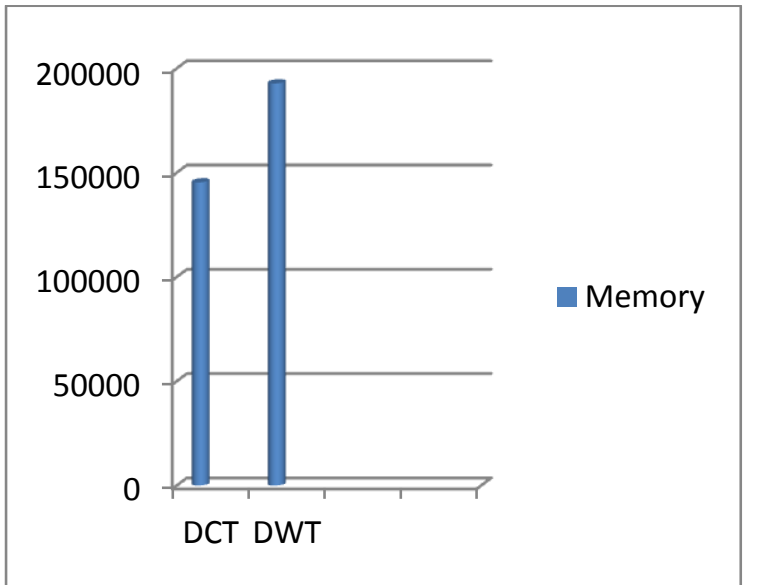

Figure 1.8 memory utilization in DWT and DCT

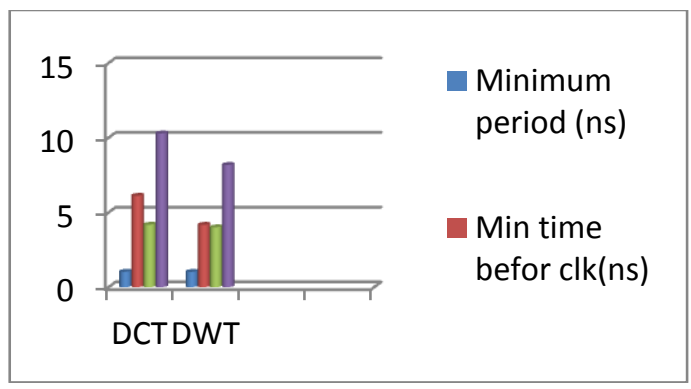

Figure 1.9 comparisons of DWT and DCT timing parameters

From the above comparison it is clear that the hardware utilization in DWT and DCT is approximately same, but if are comparing the total memory utilization in both then it is found that the memory utilization is less in DCT, it means that DWT consumes more memory in comparison as shown in figure. If there is a comparison in terms of timing parameters the minim and maximum time to reach the clock pulse at out node is less in DWT in comparison to DCT. It is analyzed that DWT is consuming $20 \%$ less time in comparison to DCT. This test is carried out for 16 x16 images. A high resolution and faster speed can be achieved using DWT when it is tested over larger images such as $1000 \mathrm{x} 1000$ pixels. The total combination path can be reduced using DWT.

\section{CONCLUSION}

The chip development of image compression techniques using Haar DWT and DCT is done successfully on Xilinx 14.2 and Modelsim 10.1b software successfully. The simulation results are tested for different test cases on images and fund successfully simulated. In this thesis considered that two image compression algorithm for image compression using VHDL, such as Discrete Wavelet Transform (DWT) and Discrete Cosine Transform (DCT). These two image compression technique DWT and DCT are compared in this thesis. The effect of these two different image compression techniques, memory utilization and timing parameters are examined. The result of DWT and DCT techniques, DWT and DCT compared by using two parameters such as memory utilization and timing utilization for image processing. It is analyzed that DWT is consuming $20 \%$ less time in comparison to DCT. So that it is observed that DWT provides a better performance in image quality at higher memory 
utilization then DCT, and also for timing utilization DWT is better than DCT for image compression. It means that DWT provide less time for image compression then DCT. So these two observations examined that DWT is better than DCT for image compression.

\section{ACKNOWLEDGEMENT}

We are truly grateful to Mr. Dinesh Kumar for providing me continuous support throughout writing a paper. His suggestion, criticism, and under lying patience so great lengths in helping me realize this survey. We are also grateful to Mr. Adesh Kumar and Mr. Om Prakash Verma, for this encouragement and suggestion during preparation. We are also thank for IT Gopeshwar for supporting.

\section{REFERENCES}

[1] Anitha Mary and Dr .N.M. Nandhitha "VHDL design of lossy DWT based image compression technique for video conferencing" IJREAT International Journal of Research in Engineering \& Advanced Technology, Volume 1, Issue 1, March, 2013.

[2] S. Jayaraman, S. Esakkirajan, T. Veerakuma "Digital Image Processing" published by TataMcGraw Hill Education Private Limited.

[3] Harmandeep Singh Chandi and V. K. Banga "2 Dimensional Image Compression using DCT and DWT
Techniques" March 15-16, Pattaya, Thailand, IRISET ICEMCE'2013 and ICHCES'2013.

[4] Mohammed Mustafa Siddeq "Using Two Levels DWT with Limited Sequential Search Algorithm for Image Compression" Journal of Signal and Information Processing, vol. 3, pp. 51-62, February 2012.

[5] Ms. Yogita Sojitra and Prof.Abhishek Katariya "Performance Analysis of Image Compression a discrete wavelet Transform based Approach" International Journal of Scientific Engineering and Technology, Volume No.1, Issue No.3, pg:-63-66, 01 July 2012.

[6] Sonja Grgic and Mislav Grgic "Performance Analysis of Image Compression Using Wavelets".IEEE TRANSACTIONON INDUSTRIAL ELECTRONICS, VOL. 48, NO. 3, JUNE 2001

[7] Er. Kanika, Dr. Naveen Dhillon and Er. Kuldeep Sharama "Comparative Analysis of Discrete Wavelet Transform and Fast Wavelet Transform on Image compression". International Journal of Engineering Research \& Technology (IJERT). Vol. 1 Issue 5, July 2012.

[8] Nivedita, Pradeep Singh and Sonika Jindal "A Comparative Study of DCT and DWT-SPIHT" IJCEM International Journal of Computational Engineering \& Management, Vol. 15 Issue 2, March 2012. 